\title{
Light chain deposition disease in kidney: A review of the literature
}

\author{
Pant $\mathrm{AD}^{1}$, Solez $\mathrm{K}^{2}$ \\ ${ }^{I}$ Department of Pathology, Tribhuvan University Teaching Hospital, Kathmandu, Nepal. \\ ${ }^{2}$ Department of Laboratory Medicine and Pathology, University of Alberta, Canada
}

\section{Keywords: \\ Light chain deposition disease; \\ Renal failure; \\ Plasma cell dyscrasia; Immunofluroescence; \\ Electron microscopy}

\begin{abstract}
Light chain deposition disease is a rather uncommon monoclonal gammopathy with predominantly renal manifestations with presence of monoclonal light chains in serum and urine. It usually occurs in elderly male patients but can have a wide age range. Patients usually present with proteinuria and some renal dysfunction, but other organs like the liver and heart may also be involved and lead to the death of the patient. The survival of the patients is only about 18 months and benefit of renal transplantation in these patients is debatable, because of high chances of recurrence. Typical light microscopic diagnostic features are important to recognize this disease, which include mesangial nodules and thickening of tubular and glomerular basement membranes. Immunofluorescence shows a monoclonal pattern for kappa light chain, or much less commonly lambda light chain. Electron microscopy is further invaluable in identifying the granular deposits in the mesangial nodules and basement membranes.
\end{abstract}

\section{INTRODUCTION}

Light chain deposition disease (LCDD) is a rather uncommon monoclonal gammopathy. It is a systemic disease with cardiac, hepatic, and gastrointestinal lesions, with kidney impairment that usually dominates the picture. ${ }^{1,2}$ The patients with renal involvement usually present with nephrotic syndrome or asymptomatic proteinuria with renal impairment, which may be rapidly progressive in some cases.

In LCDD, the light chain fragments do not form amyloid fibrils; hence they are also known as non-amyloid immunoglobulin light chains. ${ }^{2}$ These light chains are overproduced by an abnormal clone of B cells, usually seen in plasma cell dyscrasias and lymphoproliferative disorders. The disease may occur in absence of any hematological

\section{Correspondence:}

Dr. Anil Dev Pant, $M D$

Department of Pathology, T.U. Teaching Hospital, Kathmandu, Nepal.

E-mail:adpant@yahoo.com disorder, despite an extended follow-up period. These cases are known as idiopathic LCDD. ${ }^{3}$

The light chains produced are filtered by the glomeruli and reabsorbed in the proximal tubules via receptor mediated endocytosis; hence, the kidney is a prominent target for the deposition of these light chains. ${ }^{3}$

It is a difficult condition to diagnose because the patients may not have a known lymphoplasmacytic disorder, and in $40 \%$ of cases, there may not be one. If monoclonal light chains are present in serum and/or urine, a diagnosis is possible.

In the study by Paueksakon et al, none of the patients with LCDD had multiple myeloma, though all of them had some degree of bone marrow plasmacytosis. ${ }^{4}$

Other lesions that occur due to deposition of monoclonal light chains include Bence-Jones cast nephropathy and amyloid 
(light chain associated). Of these, only cast nephropathy is regularly associated with multiple myeloma. These lesions usually occur alone, but may occur in combination. ${ }^{5}$

We take a more detailed look at all the morphological features, immunofluorescence findings, electron micrographs, and possible diagnostic pitfalls of this complicated condition.

\section{DISCUSSION}

\section{Historical considerations}

In 1845 , a prominent physician in London, Dr. Thomas Watson examined a patient who presented with severe weakness, puffiness of face and ankles, and pallor. His urine was described as opaque, acidic and having high specific gravity and sent to Dr. Henry Bence Jones, a famous chemical pathologist at that time. Dr. Watson believed that the high specific gravity and opacity of urine was due to presence of an abnormal proteinaceous substance. This protein was later identified as Bence Jones protein. These proteins were proposed to be toxic by Krauss in the early 1920s. Korngold and Lipari further designated the types of Bence Jones proteins as kappa and lambda. These were found to be identical to light chains prepared from serum immunoglobulin $G$ myeloma protein, by Edelman and Gally. ${ }^{6}$

Heavy chain deposition disease was described in 1993 by Aucouturier et al. Though there were similarities with light chain deposition disease, it was finally recognized as a separate entity. ${ }^{7}$

Currently, the term Monoclonal Immunoglobulin Deposition Disease is used to encompass the different deposition diseases. They include light LCDD, heavy chain deposition disease, and light chain cast nephropathy.

\section{Biochemical findings, clinical course and therapy}

Patients are typically elderly with a slight male preponderance. However, a wide range of patients ranging from 28 to 94 years were found in a relatively large study performed by Pozzi et al. ${ }^{3}$ Most patients also have monoclonal proteins in serum and urine. Patients can present with acute renal failure, rapidly progressive renal failure or chronic renal failure. Other less common features include hypertension and hematuria. Deposition in other rarer sites includes lung, brain, skin and peripheral nerves.

Proteinuria is usually $>1 \mathrm{~g} / \mathrm{dl}$ and occurs in $80 \%$ to $90 \%$ of patients. It is nephrotic in 28 to $53 \%$ of cases; the proteinuria is non-selective. Hematuria may also be seen in up to $30 \%$ of the cases. ${ }^{7}$

Monoclonal light chains may appear only intermittently at low concentrations in serum and/or urine. Immunofixation and high-resolution electrophoresis are much more sensitive than traditional electrophoretic methods. ${ }^{7} \mathrm{Up}$ to $15 \%$ of cases may not show abnormality in immunoelectrophoresis. ${ }^{8}$

Median survival time from diagnosis is about 18 months. Early chemotherapy may slow progression of chronic renal failure and prolong life. ${ }^{9}$ Survival is variable, and ranges from 1 month to 10 years from the onset of symptoms. Death usually occurs due to heart or liver failure or from its progression to multiple myeloma. ${ }^{2}$ However, there has been a report of reversal of nodular lesions and renal function after chemotherapy. ${ }^{10}$

Renal transplantation in these patients has been seriously debated. Some suggest that the patient should be free of extra-renal manifestations in the initial presentation or for more than one year in patients on chemotherapy for plasma cell dyscrasia. These patients represents a minority of the cases. ${ }^{11}$ Recurrence in the allograft is quite common as discovered by Leung in 5 out of 7 cases. $^{8}$ The only long term survivor was a patient who had a normal bone marrow biopsy before the transplantation. ${ }^{12}$ Recurrence has been estimated to be more than $50 \%$ within 4 years after transplantation. Conversely, de novo LCDD rarely occurs in allografts. ${ }^{13}$

Factors indicating poorer survival include the initial creatinine and serum calcium levels, types of immunoglobulin deposits and types of lesions seen in the renal biopsy. ${ }^{8}$

\section{Light microscopy and differential diagnosis}

Microscopically, all the different renal compartments may be affected. The glomeruli may appear unremarkable in the early part of the disease. In later cases, there may be increased mesangial matrix in the glomeruli, along with diffuse and nodular glomerulosclerosis. These nodules should be differentiated from other forms of renal disease which show nodular glomerulosclerosis, like diabetes, amyloidosis and cryoglobulinemia (fig. 1A and B)

Nodular glomerulosclerosis may be seen in $50 \%$ of cases with nephrotic range proteinuria and $25 \%$ of non-nephrotic range patients. ${ }^{9}$ A common finding is the thickening and wrinkling of peripheral basement membranes (fig. 2). ${ }^{3}$ Crescents and membranoproliferative pattern with lobular accentuation and hypercellularity may also be seen. ${ }^{8}$

Tubules show thickening of tubular basement membranes, and there may be extensive tubular atrophy and interstitial fibrosis (fig. 2). Arteriolar hyalinosis may be one of the findings and there may also be concentric thickening of small and medium sized arteries. ${ }^{7}$

Patients with predominant glomerular deposition of light chains usually develop nephrotic syndrome, while those 
with tubular deposition develop renal failure and mild proteinuria. $^{2}$

These deposits are negative for Congo Red, an important differentiating point from amyloidosis., ${ }^{2,8}$

LCDD usually occurs singly, without presence of any associated diseases. However, Lam and Chan reported an unusual case with there was LCDD, renal amyloidosis and cast nephropathy. ${ }^{14}$ Other associations seen in a series of 24 cases were with cast nephropathy with light chain disease and light chain disease with amyloidosis of other organs (tongue and heart). ${ }^{15}$ LCDD has also been known to occur in conjunction with heavy chain disease. ${ }^{7}$

\section{Immunofluorescence findings}

Immunofluorescence typically shows a monoclonal linear staining in the glomerular and tubular basement membrane, mostly for kappa (fig. 3A). Because light microscopy may be unremarkable, immunofluorescence is a must in order to rule out light chain disease, especially in patients with suspected disease. $^{3}$

Sometimes, the typical linear staining pattern may not be present especially when there is a small amount of deposition of light chains. In these cases, electron microscopy will be more sensitive. ${ }^{16}$ In addition, generic antibodies to kappa and lambda light chains may not detect all light chains which may have structural differences or may be altered in presence of coexisting diabetes (fig 3B). They can be detected only by specific antibodies directed towards these altered light chains. ${ }^{7}$

\section{Electron microscopy findings}

Electron microscopy shows nodular expansion of mesangial matrix. Granular electron dense deposits can be seen in the mesangium and renal basement membranes (fig.4). ${ }^{9}$ Even immunofluorescence negative cases can be diagnosed with the help of electron microscopy, which has a much higher resolution and is able to detect even small deposits.

Because the electron microscopy samples are small,
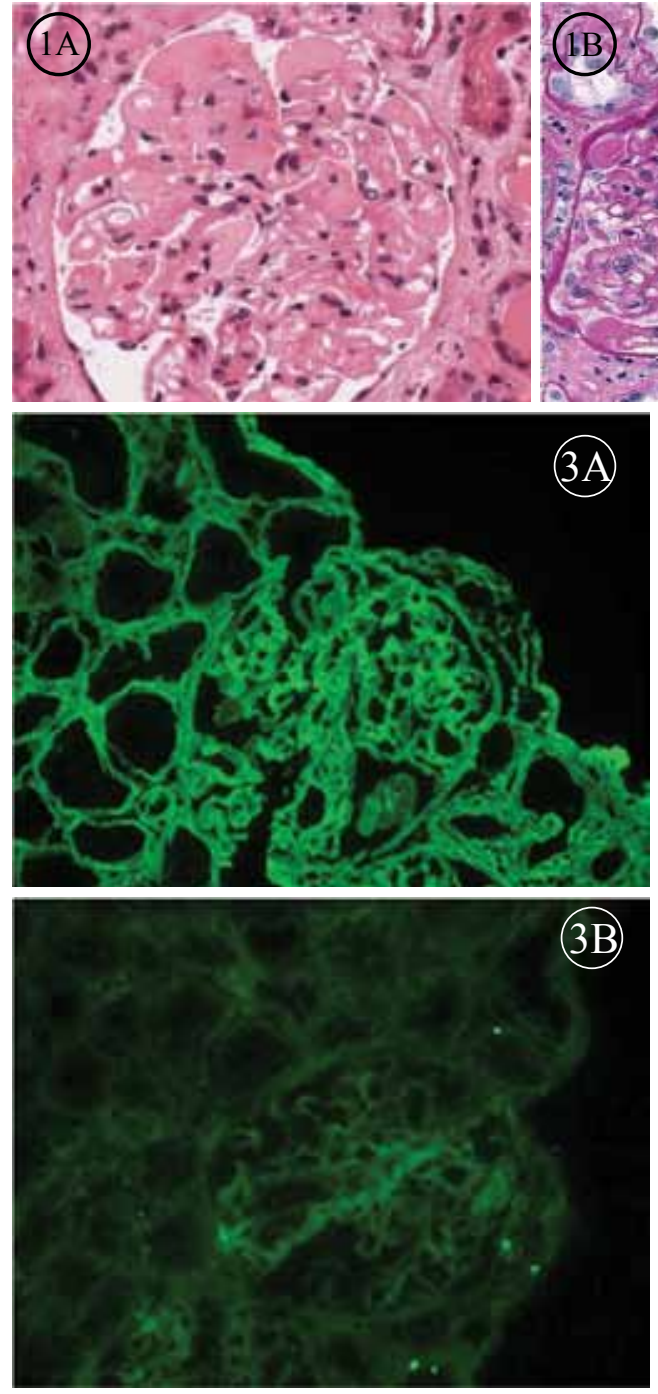
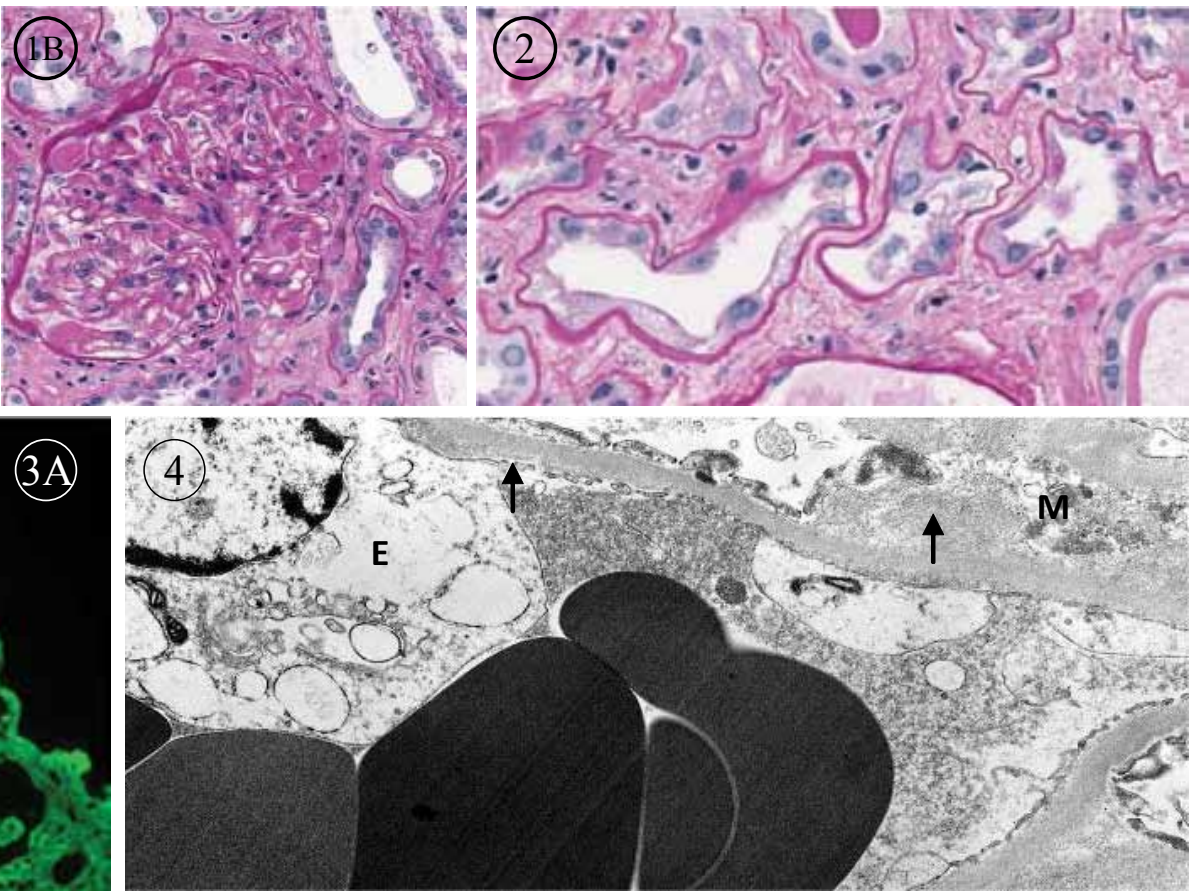

Figure 1A: Section showing nodules in mesangium which resembles nodules of diabetic glomerulosclerosis (HE, X40).

Figure 1B: The stain highlights the nodules and also shows thickening of glomerular and surrounding tubular basement membranes (PAS Stain, X20).

Figure 2: Extensive tubular basement membrane wrinkling and multilayering in areas of tubular atrophy and fibrosis (PAS Stain, X40).

Figure 3A: Immunofluorescence findings showing bright positive staining in the glomeruli and tubular basement membranes for kappa light chain.

Figure 3B: Immunofluorescence staining lambda light chains is negative in the same glomerulus, thus confirming the monoclonality of the light chains

Figure 4: Transmission electron microscopy showing presence of deposits in the mesangium and capillary basement membrane (arrows). E=Endothelial cell, $M=$ mesangium, $C=$ capillary lumen 
the lesions may be missed, causing discrepancy with the immunofluorescence findings. ${ }^{3,16}$ Either immunofluorescence or electron microscopy can be more diagnostic than the other, due to limitations of each investigation.

Ultrastructural immunolabeling may be even more helpful, and combines immunofluorescence and ultrastructure into a single diagnostic tool. This may be invaluable, especially in early and subtle lesions with light chain deposition in areas other than the mesangial nodules. ${ }^{16,17}$

\section{CONCLUSION}

LCDD should be considered as a possibility in any patients with renal insufficiency of unknown origin. In patients with nephrotic syndrome or chronic renal failure, LCDD should be considered in the differential diagnosis, especially if there is nodular glomerulosclerosis and thickening of tubular basement membranes in the renal biopsy. The background of a lymphoplasmacytic disorder makes this diagnosis all the more likely. Repeated testing with urine and serum for light chains, bone marrow examination, and detailed renal biopsy workup along with light chain immunofluorescence and electron microscopy is required in order to diagnose this elusive disorder.

\section{REFERENCES}

1. Salant JS, Sanchorwala V, D'Agati VD. A case of atypical light chain deposition disease - diagnosis and treatment. Clin J Am Soc Nephrol 2007;2:858-67.

2. Fabbian F, Stabellini N, Sartori S et al. Light chain deposition disease presenting as paroxysmal atrial fibrillation: a case report. J Med Case Reports 2007;1:1-4.

3. Pozzi C, D’Amico M, Fogazzi GB. Light chain deposition disease with renal involvement: clinical characteristics and prognostic factors. Am J Kidney Dis 2003;42:1154-63.
4. Paueksakon P, Revelo MP, Fogo AB et al. Monoclonal gammopathy: significance and possible causality in renal disease. Am J Kidney Dis 2003;42:87-95.

5. Cohen AH. The Kidney in plasma cell dyscrasias: Bence-Jones cast nephropathy and light chain deposit disease. Am J Kidney Dis 1998;32:529-32.

6. Herrera GA, Picken MM. Renal diseases associated with plasma cell dyscrasias, amyloidoses, waldenstrom macroglobulinemia, and cryoglobulinemic nephropathies. In: Jennetts JC, Olson JL, Schwartz MM, Silva FG, eds. Hepinstall's Pathology of the Kidney. Lippincott Williams and Wilkins; 2007. PP 854-902.

7. Herrera GA. Renal Manifestations of Plasma Cell Dyscrasias: An appraisal from the Patient's Bedside to the Research Laboratory. Ann Diagn Pathol 2000;4:174-200.

8. Leung N, Lager DJ, Fervenza FC et al. Long-term outcome of renal transplantation in light-chain deposition disease. Am J Kidney Dis 2004;43:147-53.

9. Hall CL, Peat DS. Light chain deposit disease: a frequent cause of diagnostic difficulty. Nephrol Dial Transplant 2001;16:1939-41.

10. Komatsuda A, Wakui H, Ohtani H. Disappearance of nodular mesangial lesions in a patient with light chain nephropathy after long-term chemotherapy. Am J Kidney Dis 2000;35:1-5.

11. Leung N, Rajkumar SV. Renal Manifestations of Plasma Cell Disorders. Am J Kidney Dis 2007;50:155-65.

12. Heher EC, Spitzer TR, Goes NB. Light chains: heavy burden in kidney transplantation. Transplantation 2009;87:947-52.

13. Taneda S, Honda K, Horita S et al. Light chain deposition disease after renal transplantation. Am J Kidney Dis 2008;52: 621-5.

14. Qian Q, Leung N, Sethi S et al. Coexistence of myeloma cast nephropathy, light chain deposition disease, and nonamyloid fibrils in a patient with multiple myeloma. Am J Kidney Dis 2010;56: 971-6.

15. Strom EH, Fogazzi GB, Mihatsch MJ, et al. Light chain deposition disease of the kidney. Morphological aspects in 24 patients. Virchows Archiv 1994;425:271-80.

16. Herrera GA. The contributions of electron microscopy to the understanding and diagnosis of plasma cell dyscrasia-related renal lesions. Med Electron Microsc 2001;34:1-18.

17. Santostefano M, Zanchelli F, Zaccaria A, Polleti G, Fusaroli M. The ultrastructural basis of renal pathology in monoclonal gammopathies. J Nephrol 2005;18:659-75. 